\title{
On Dihedral Flows of Generalized Petersen Graphs Cellularly Embedded in Closed Orientable Surfaces
}

\author{
Francis E. Yap, \\ Tjaart Jan B. Estrada, Priscilla S. Macansantos
}

\begin{abstract}
Let $G$ be a graph and $S$ be a closed orientable surface. Litjens defined $G$ to have a nowhere-identity dihedral 4-flow if for each $S$ in which $G$ can be cellularly embedded, there is a rotation system $\pi$ of $G$ which gives a nowhere-identity dihedral 4-flow [4]. Furthermore, he proved the existence of nowhere-identity dihedral 4flows for bridgeless cubic graphs with 16 or less vertices. We give a method for exhibiting the nowhere-identity dihedral $n$-flows in generalized Petersen graphs and illustrate the results by exhibiting the nowhere-identity dihedral 4 -flows of $G(3,1)$.
\end{abstract}

2000 AMS Subject Classification: 05C10, 05C21, 05C50.

Keywords and Phrases: dihedral flow, generalized Petersen graph, embedded graph. This research was supported by Accelerated Science and Technology Human Resource Development Program - National Science Consortium of the Department of Science and Technology - Science Education Institute. 


\section{Introduction}

Let

$$
\mathbb{D}=\left\{\left[\begin{array}{cc} 
\pm 1 & a \\
0 & 1
\end{array}\right]: a \in \mathbb{Z}\right\}
$$

and let

$$
\mathbb{D}^{<n}=\left\{\left[\begin{array}{cc} 
\pm 1 & a \\
0 & 1
\end{array}\right]: a \in \mathbb{Z},|a|<n, n \in \mathbb{Z}^{+}\right\}
$$

Let $G=(V, E)$ be a graph. A nowhere-identity $\mathbb{D}$-flow is an assignment of non-identity elements from $\mathbb{D}$ to the edges of $G$ such that, for each $v \in V$, the product of the elements assigned to the edges incident to $v$ is equal to the identity. A nowhere-identity dihedral $n$-flow is a nowhereidentity $\mathbb{D}$-flow with elements restricted to $\mathbb{D}^{<n}$. In both cases, a given cyclic order of the edges incident to each vertex removes any ambiguity regarding the product of the assigned elements.

Elements of $\mathbb{D}$ of the form

$$
\left\{\left[\begin{array}{ll}
1 & i \\
0 & 1
\end{array}\right] \mid i \in \mathbb{Z}\right\}
$$

are called rotations while elements of the form

$$
\left\{\left[\begin{array}{cc}
-1 & i \\
0 & 1
\end{array}\right] \mid i \in \mathbb{Z}\right\}
$$

are called reflections.

The collection $\pi=\left\{\pi_{v} \mid v \in V\right\}$ of cyclic orders of $G$ is called the rotation system of $G$. The following theorem is due to Heffter, Edmonds, and Ringel [5]:

Theorem 1.1 (Heffter-Edmonds-Ringel Rotation Principle). The set of all rotation systems $\pi$ of a graph $G$ is in a one-to-one correspondence, up to homeomorphism, with the set of all cellular embeddings of $G$ in a closed orientable surface $S$. 
Litjens defined in [4] that $G$ has a nowhere-identity dihedral 4-flow if for each closed orientable surface $S$ in which $G$ can be cellularly embedded, there is a rotation system $\pi$ of $G$ which gives a nowhere-identity dihedral 4-flow. With this definition, he showed that:

Theorem 1.2. There exists a nowhere-identity dihedral 4-flow for the Petersen graph.

He furthermore proved that this also holds for a superset of graphs containing the Petersen graph.

Theorem 1.3. There exist nowhere-identity dihedral 4-flows for bridgeless cubic graphs with 16 or less vertices.

In relation to the above-mentioned results, we provide a method for exhibiting the nowhere-identity dihedral $n$-flows of generalized Petersen graphs and illustrate the results by exhibiting the nowhere-identity dihedral 4-flows of $G(3,1)$.

The generalized Petersen graph, $G(n, k)$, is a simple cubic graph consisting of $2 n$ vertices $\left\{u_{0}, u_{1}, \ldots, u_{n-1}, v_{0}, v_{1}, \ldots, v_{n-1}\right\}$ and $3 n$ edges $\left\{u_{i} u_{i+1}\right.$, $\left.u_{i} v_{i}, v_{i} v_{i+k}\right\}$ where $0 \leq i \leq n-1, n \geq 3,0<k<\frac{n}{2}$, and all subscripts are computed modulo $n$. The collection of edges $u_{i} u_{i+1}, u_{i} v_{i}$, and $v_{i} v_{i+k}$ are called the outer rim, the spokes, and the inner rim of the graph, respectively [6].

For further review of related literature that will be used throughout this research, the reader is referred to [1, 2, 3, 6] .

\section{Nowhere-Identity Dihedral $n$-Flows of $\mathbf{G}(\mathbf{n}, \mathbf{k})$}

For us to be able to find out if a graph has a nowhere-identity dihedral $n$-flow, we essentially need three things: (1) the genus of each closed orientable surface in which the graph can be cellularly embedded, (2) the equivalent graph rotation systems for each closed orientable surface, and (3) the permissible values that can be assigned to the edges of the graph to give nowhere-identity dihedral $n$-flows. 
Our strategy for determining the nowhere-identity dihedral $n$-flows of the generalized Petersen graph $G(n, k)$ is outlined and we apply it to the nowhere-identity dihedral 4-flows of the generalized Petersen graph $G(3,1)$.

1. Determine the genus range of $G(n, k)$.

The genus range of $G(3,1)$ depends on the minimum genus and maximum genus of $G(3,1)$. It can easily be shown that $\gamma_{\min }(G(3,1)) \geq 0$ and $\gamma_{\max }(G(3,1)) \leq 2$. Therefore, $G(3,1)$ can be cellularly embedded on any closed orientable surface whose genus lies in the range $\left[\gamma_{\min }(G(3,1)), \gamma_{\max }(G(3,1))\right]$.

2. Enumerate the equivalence classes of the $G(n, k)$ rotation systems.

There are $2^{6}=64$ rotation systems for $G(3,1)$ with some equivalent under rotational or reflection symmetries. We reduce this number by determining the equivalence classes. As an example, consider the graph $G(3,1)$ with rotation system

$\pi^{(1)}=\left\{\left(u_{0}^{\pi^{(1)}}, \circlearrowleft\right),\left(u_{1}^{\pi^{(1)}}, \circlearrowleft\right),\left(u_{2}^{\pi^{(1)}}, \circlearrowright\right),\left(v_{0}^{\pi^{(1)}}, \circlearrowright\right),\left(v_{1}^{\pi^{(1)}}, \circlearrowright\right),\left(v_{2}^{\pi^{(1)}}, \circlearrowleft\right)\right\}$.

The rotational symmetries of $\pi^{(1)}$ are given by the following rotation systems:

$$
\begin{aligned}
& \pi^{(2)}=\left\{\left(u_{2}^{\pi^{(2)}}, \circlearrowleft\right),\left(u_{0}^{\pi^{(2)}}, \circlearrowleft\right),\left(u_{1}^{\pi^{(2)}}, \circlearrowright\right),\left(v_{2}^{\pi^{(2)}}, \circlearrowright\right),\left(v_{0}^{\pi^{(2)}}, \circlearrowright\right),\left(v_{1}^{\pi^{(2)}}, \circlearrowleft\right)\right\} \\
& \pi^{(3)}=\left\{\left(u_{1}^{\pi^{(3)}}, \circlearrowleft\right),\left(u_{2}^{\pi^{(3)}}, \circlearrowleft\right),\left(u_{0}^{\pi^{(3)}}, \circlearrowright\right),\left(v_{1}^{\pi^{(3)}}, \circlearrowright\right),\left(v_{2}^{\pi^{(3)}}, \circlearrowright\right),\left(v_{0}^{\pi^{(3)}}, \circlearrowleft\right)\right\}
\end{aligned}
$$

Similarly, the reflection symmetries of $\pi^{(1)}$ are given by the following rotation systems:

$$
\begin{aligned}
& \pi^{(4)}=\left\{\left(u_{0}^{\pi^{(4)}}, \circlearrowright\right),\left(u_{2}^{\pi^{(4)}}, \circlearrowright\right),\left(u_{1}^{\pi^{(4)}}, \circlearrowleft\right),\left(v_{0}^{\pi^{(4)}}, \circlearrowleft\right),\left(v_{2}^{\pi^{(4)}}, \circlearrowleft\right),\left(v_{1}^{\pi^{(4)}}, \circlearrowright\right)\right\} \\
& \pi^{(5)}=\left\{\left(u_{2}^{\pi^{(5)}}, \circlearrowright\right),\left(u_{1}^{\pi^{(5)}}, \circlearrowright\right),\left(u_{0}^{\pi^{(5)}}, \circlearrowleft\right),\left(v_{2}^{\pi^{(5)}}, \circlearrowleft\right),\left(v_{1}^{\pi^{(5)}}, \circlearrowleft\right),\left(v_{0}^{\pi^{(5)}}, \circlearrowright\right)\right\} \\
& \pi^{(6)}=\left\{\left(u_{1}^{\pi^{(6)}}, \circlearrowright\right),\left(u_{0}^{\pi^{(6)}}, \circlearrowright\right),\left(u_{2}^{\pi^{(6)}}, \circlearrowleft\right),\left(v_{1}^{\pi^{(6)}}, \circlearrowleft\right),\left(v_{0}^{\pi^{(6)}}, \circlearrowleft\right),\left(v_{2}^{\pi^{(6)}}, \circlearrowright\right)\right\}
\end{aligned}
$$

These six rotation systems form an equivalence class whose elements are equivalent under a rotational or reflection symmetry. We repeat this process by selecting one rotation system that is still not 
a member of any equivalence class and determining its rotational and reflection symmetries. The process terminates if every rotation system is a member of an equivalence class. For $G(3,1)$, we are able to identify 12 equivalence classes representing the distinct rotation systems of $G(3,1)$.

3. For each $G(n, k)$ rotation system equivalence class, compute the corresponding genus of the closed orientable surface.

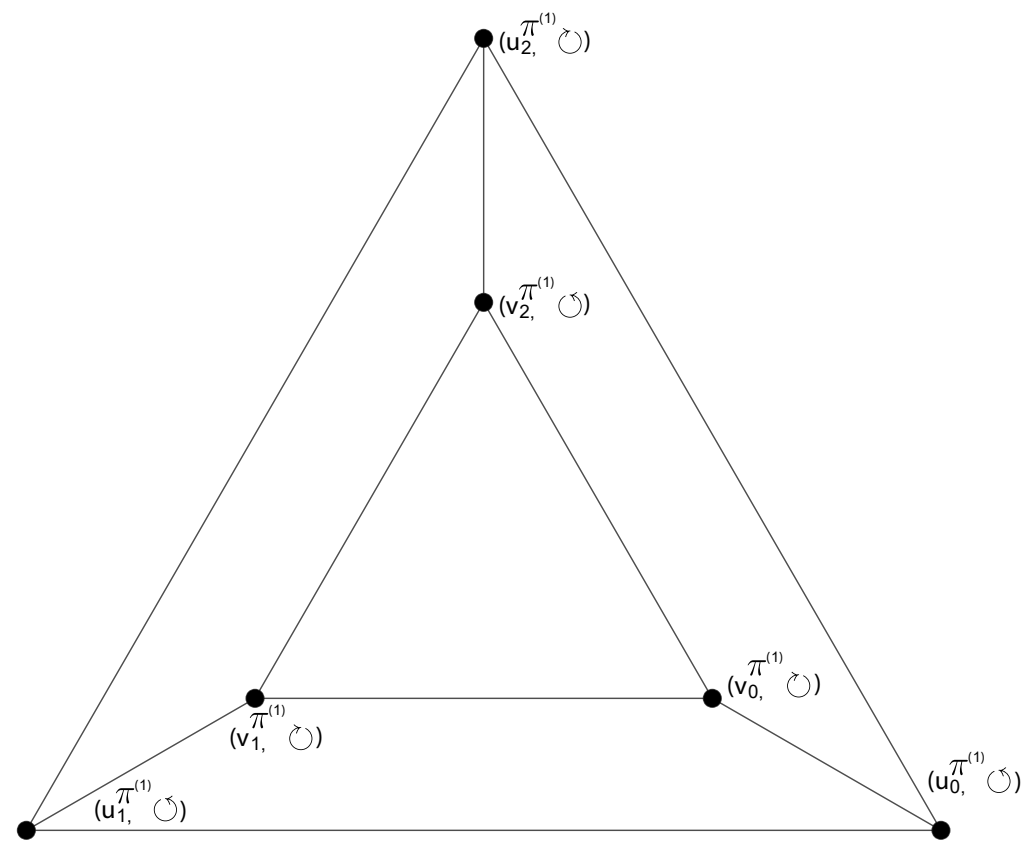

Figure 1: $G(3,1)$ rotation system $\pi^{(1)}$.

Consider the $G(3,1)$ rotation system equivalence class represented by $\pi^{(1)}$ shown in Figure 1. This graph rotation system is equivalent to a cellular embedding of the graph in a closed orientable surface. We use the face tracing algorithm to enumerate and count the number of faces in the embedding. The unique closed walks representing 
the boundary of the faces are given in the following:

- $\left(u_{0}^{\pi^{(1)}}, u_{1}^{\pi^{(1)}}, v_{1}^{\pi^{(1)}}, v_{2}^{\pi^{(1)}}, v_{0}^{\pi^{(1)}}, u_{0}^{\pi^{(1)}}\right)$

- $\left(u_{0}^{\pi^{(1)}}, u_{2}^{\pi^{(1)}}, v_{2}^{\pi^{(1)}}, v_{1}^{\pi^{(1)}}, v_{0}^{\pi^{(1)}}, v_{2}^{\pi^{(1)}}, u_{2}^{\pi^{(1)}}, u_{1}^{\pi^{(1)}}, u_{0}^{\pi^{(1)}}\right)$

- $\left(u_{0}^{\pi^{(1)}}, v_{0}^{\pi^{(1)}}, v_{1}^{\pi^{(1)}}, u_{1}^{\pi^{(1)}}, u_{2}^{\pi^{(1)}}, u_{0}^{\pi^{(1)}}\right)$

We listed 3 walks with 2 walks having 5 oriented edges each and one walk having 8 oriented edges for a total of 18 oriented edges. Moreover, the 18 oriented edges in the list are also distinct. Since this also happens to be the number of oriented edges of $G(3,1)$, we know that the face tracing algorithm [5] gives us the desired result. Therefore, $G(3,1)$ has 3 faces.

This graph rotation system is equivalent to a cellular embedding of the graph in a closed orientable surface whose genus is equal to 1 by Euler's Formula.

The same process was applied to all the graph rotation system equivalence classes. The results showed that $G(3,1)$ can be cellularly embedded in 3 closed orientable surfaces. The genus of these surfaces are $\gamma \in\{0,1,2\}$.

4. List all the perfect matchings of $G(n, k)$. These matchings represent the edges that yield rotations.
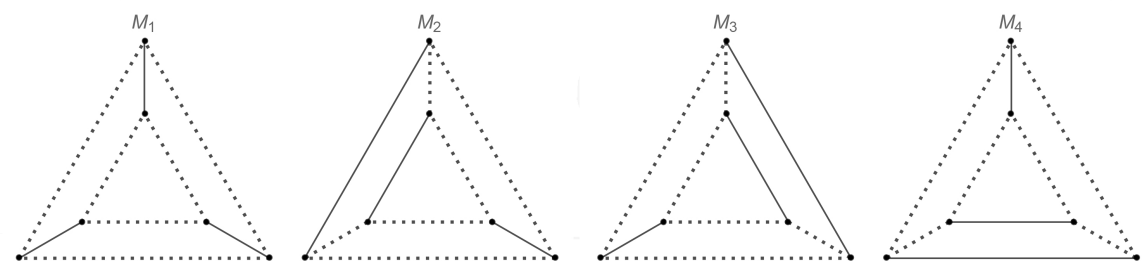

Figure 2: Perfect matchings of $G(3,1)$.

All perfect matchings of $G(3,1)$ are shown in Figure 2, The matching edges are depicted by solid line and yield rotations, while the 
remaining edges are depicted by dotted lines and yield reflections.

5. Select a $G(n, k)$ rotation system and a perfect matching of the graph to solve the nowhere-identity dihedral $n$-flow. A graph has a nowhere-identity dihedral $n$-flow if a solution exists for every genus of the graph.

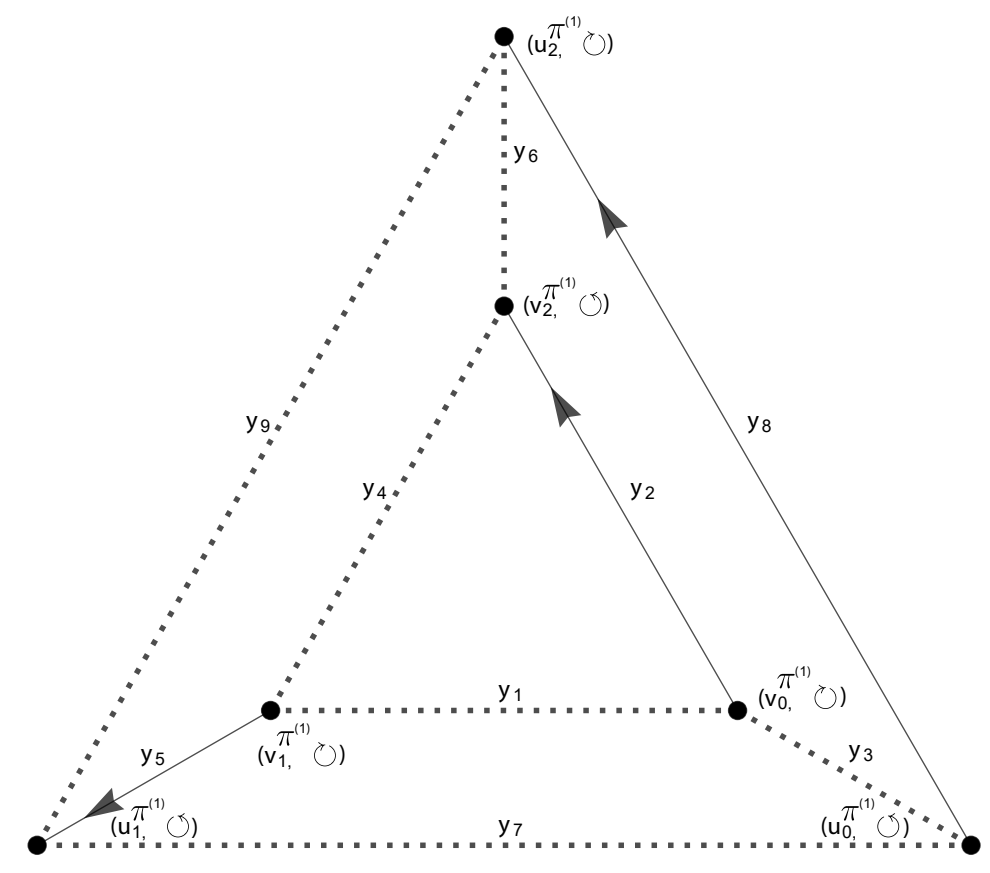

Figure 3: $G(3,1)$ rotation system $\pi^{(1)}$ and perfect matching $M_{3}$.

The generalized Petersen graph $G(3,1)$ can be cellularly embedded in 3 closed orientable surfaces. Moreover, it has 12 rotation system equivalence classes and 4 perfect matchings. This means that proving the existence of nowhere-identity dihedral 4-flows of $G(3,1)$ involves 3 up to 48 computations.

To illustrate one computation, we select the $G(3,1)$ rotation system $\pi^{(1)}$ and the perfect matching $M_{3}$. As shown in Figure 3 , we also 
added arrowheads of arbitrary direction and variables for the edge values.

For each vertex, we take into consideration the cyclic order and the orientation of the arrowhead relative to the vertex and arrive at the equations

$$
\begin{array}{rrr}
-y_{2}+y_{3}=y_{1} & -y_{8}+y_{7}=y_{3} \\
-y_{5}+y_{4}=y_{1} & y_{5}+y_{9}=y_{7} \\
y_{2}+y_{6}=y_{4} & y_{8}+y_{6}=y_{9} .
\end{array}
$$

Note that the domain of $y_{i}$ is $\mathbb{Z}$ for $1 \leq i \leq 9$ subject to the constraint $0 \leq\left|y_{1}\right|,\left|y_{3}\right|,\left|y_{4}\right|,\left|y_{6}\right|,\left|y_{7}\right|,\left|y_{9}\right|<4$ and $0<\left|y_{2}\right|,\left|y_{5}\right|,\left|y_{8}\right|<4$. A computer-aided implementation of this problem yielded 108 solutions representing the number of ways to exhibit nowhere-identity dihedral 4-flows for the given pair of rotation system and perfect matching of $G(3,1)$. Out of the 108 solutions, 32 are nowhereidentity dihedral 3-flows. Four (4) out of the 32 solutions are nowhereidentity dihedral 2-flows. A solution that is a nowhere-identity dihedral 2-flow, which consequently is also a nowhere-identity dihedral 3 -flow and a nowhere-identity dihedral 4 -flow, is given by

$$
\left[\begin{array}{lllllllll}
y_{1} & y_{2} & y_{3} & y_{4} & y_{5} & y_{6} & y_{7} & y_{8} & y_{9}
\end{array}\right]^{T}=\left[\begin{array}{lllllllll}
-1 & 1 & 0 & 0 & 1 & -1 & 1 & 1 & 0
\end{array}\right]^{T} .
$$

\section{Conclusions}

We introduce a concept called the rotation number of a rotation system. Denote by $\rho_{\circlearrowright}$ and $\rho_{\circlearrowleft}$ the number of vertices of $G$ with clockwise and counterclockwise cyclic orders, respectively. We define the rotation number of $G$ as $\rho=\min \left(\rho_{\circlearrowright}, \rho_{\circlearrowleft}\right)$. From the exhaustive analysis of $G(3,1)$ we have the following observations:

1. If $\rho=0$, then solutions can be found for every perfect matching of the graph. 
2. If $\rho=1$, then no solution can be found for any perfect matching of the graph.

3. If $\rho=2$, then solutions can be found for every perfect matching of $G(3,1)$ except for one perfect matching consisting of spokes of $G(3,1)$. 


\section{References}

[1] G. Chartrand and P. Zhang, Introduction to Graph Theory, McGrawHill (2005).

[2] M. DeVos, Flows on Graphs, Princeton University (2000).

[3] R. Diestel, Graph Theory, Springer Nature (2017).

[4] B. Litjens, On dihedral flows in embedded graphs, Journal of Graph Theory 91 (2018), no. 2, 174-191.

[5] B. Mohar and C. Thomassen, Graphs on Surfaces, The John Hopkins University Press (2001).

[6] M. Watkins, A Theorem on Tait Colorings with an Application to the Generalized Petersen Graphs, Journal of Combinatorial Theory 6 (1969), no. 2, 152-164.

Francis E. Yap

University of the Philippines

Baguio

Department of Mathematics and

Computer Science

feyap@up.edu.ph
Tjaart Jan B. Estrada

Don Mariano Marcos Memorial State University

Mathematics and Allied Disciplines Department tjestrada@dmmmsu.edu.ph

Priscilla S. Macansantos

University of the Philippines

Baguio

Department of Mathematics and

Computer Science

psmacansantos@up.edu.ph 